\title{
Expression of Keratinocyte Growth Factor in Periapical Lesions
}

\author{
Z. Gao' ${ }^{1}$, C.M. Flaitz ${ }^{1}$, and I.C. Mackenzie ${ }^{2 *}$ \\ ${ }^{1}$ Dental Branch, University of Texas, Houston Health Science Center; and ${ }^{2}$ Department of Cariology, School of Dentistry, University of \\ Michigan, 1011 N. University, Ann Arbor, Michigan 48109-1078; * to whom correspondence should be addressed
}

\begin{abstract}
The epithelial proliferation associated with inflammatory periapical lesions and with periapical cyst formation represents an interesting but poorly understood pathological change. Keratinocyte growth factor (KGF) is a recently identified growth factor that is produced by stromal fibroblasts and acts specifically to stimulate epithelial growth and differentiation. To investigate its possible role in the activation of the normally quiescent rests of Malassez, we examined the expression of KGF by in situ hybridization of sections of normal periodontal ligament (PDL) and of 12 periapical granulomas or cysts. Normal PDL and periapical granulomas with scant inflammatory infiltration showed few cells expressing message for KGF. However, KGFexpressing cells were found in the connective tissue stroma close to dense foci of inflammatory cells and to proliferating epithelial elements and cystic epithelial linings. Examination of tissues by the reverse-transcription polymerase chain reaction (RT-PCR) showed KGF expression in 4 specimens of periapical lesions but low or undetectable levels in normal PDL. These observations suggest that the induction of KGF expression in the stromal cells of periapical lesions may play an important role in stimulating the epithelial proliferation associated with cyst formation.
\end{abstract}

Key words: keratinocyte growth factor, periapical lesions, inflammatory cysts, RT/PCR, in situ hybridization.

Received May 22, 1995; Accepted May 7, 1996

\section{Introduction}

The epithelial lining of periapical cysts is generally considered to be derived from proliferation of the epithelial rests of Malassez (Ten Cate, 1972; Shear, 1985), but the factors responsible for the activation of these normally quiescent rests are poorly understood. The changing patterns of expression of epithelial cytokeratins found during the early stages of cyst formation in inflammatory periapical lesions lend support to the general hypothesis of activation of the rests to a new phenotypic pattern (Gao et al., 1988a). Some form of alteration in the connective tissue environment supporting the cell rests is thought to be responsible for this activation (Ten Cate, 1972). Local changes in tissue $\mathrm{pH}$ or carbon dioxide tension have been suggested (Grupe et al., 1967), and it is apparent that epithelial proliferation is typically associated with inflammation and the local accumulation of various types of immune cells (Stern et al., 1982; Cymerman et al., 1984; Torabinejad and Kettering, 1985; Gao et al., 1988b). Recent work indicates that tissue concentrations of growth factors are markedly affected by inflammation, due both to the cytokines produced by the inflammatory cells themselves and to the effects of these cytokines on the local stromal cells (Luger and Schwartz, 1994). Growth factors are important mediators of intercellular communication between connective tissues and epithelium; information about the growth factors that are generated in periapical lesions may therefore assist in our understanding of the mechanisms of stimulation of epithelial proliferation and subsequent cyst formation.

Several growth factors have stimulatory effects on epithelial proliferation and differentiation. The recently described keratinocyte growth factor (KGF) appears to be of particular interest. KGF is a stromally derived growth factor with stimulatory paracrine actions specifically targeted to epithelial cells (Finch et al., 1989; Housley et al., 1994; Imagawa et al., 1994; Yi et al., 1994). KGF production is markedly up-regulated during epithelial wound healing (Werner et al., 1992, 1994), and there is increasing evidence that KGF expression by stromal fibroblasts is associated 
with the maintenance of normal epithelial structure (Marchese et al., 1990). Little detailed information is yet arailable about the expression of KGF in oral tissues. It appears to be expressed by fibroblasts beneath the mucosal epithelium but not by those deeper in the submucosa or periodontal ligament (Gao and Mackenzie, 1996a, b). It is possible that quiescence of the rests of Malassez is normally associated with low local levels of KGF or related growth factors in the PDL but that inflammation enhances the expression of growth factors necessary for their activation.

The aim of the present study was to explore the potential role of KGF in the initiation and promotion of epithelial growth during periapical cyst formation by comparing the pattern of KGF expression in various periapical lesions with that of normal periodontal ligament using the techniques of in situ hybridization and RT-PCR.

\section{Materials and methods}

\section{Specimens for in situ hybridization}

The material used consisted mainly of archival blocks of waxembedded biopsies that had been received by a surgical oral pathology laboratory during the previous 12 months. All specimens had been fixed in $10 \%$ formalin and processed for routine histopathologic examination. The 12 specimens selected for the study had been previously diagnosed as periapical lesions and included 6 periapical granulomas and 6 periapical cysts. Additional samples of normal PDL were collected by dissection from the roots of extracted vital teeth and were similarly processed. Sections were cut at $6-\mu \mathrm{m}$ intervals and collected on Probeplus slides (Fisher).

\section{Specimens for RT-PCR}

Tissue was obtained from 4 periapical lesions during apicoectomy ( 2 samples) or from teeth that were extracted with inflamed periapical soft tissue attached to the roots (2 samples). Samples of normal PDL were similarly removed from the apical root surfaces of extracted teeth which showed no evidence of periodontal or periapical pathosis. Half of each sample was fixed in $10 \%$ formalin for histological evaluation and the other half placed into cold tissue culture medium (DMEM, Sigma) at $4^{\circ} \mathrm{C}$ and rapidly transferred to the laboratory for RNA extraction. For RNA analysis, tissues were homogenized, and total RNA was extracted by the RNA Stat 60 method (Lawrence, Newark, NJ). The RNA yield was quantitated by spectrophotometric measurement of absorption at 260 and $280 \mathrm{~nm}$, and RNA samples were kept at $-90^{\circ} \mathrm{C}$ until used for RT-PCR. All samples were collected in accordance with a protocol approved by the Committee for the Protection of Human Subjects at the University of Texas.

\section{In situ hybridization}

The general methods used for demonstration of KGF expression by in situ hybridization were as previously described in detail elsewhere (Gao and Mackenzie, 1996b). For riboprobe preparation, plasmid containing a 496-bp fragment of KGF cDNA was linearized with appropriate restriction endonucleases as templates. Sense and antisense probes were synthesized with digoxigenin-11-UTP (Boehringer Mannheim, Indianapolis, IN) by in vitro transcription. The yield of probes was quantitated against supplied standards. Paraffin sections were deparaffinized by treatment in xylene and rehydrated in graded alcohols prior to treatment with proteinase-K for 30 $\min$ at $37^{\circ} \mathrm{C}(2 \mu \mathrm{g} / \mathrm{mL})$. Sections were then hybridized in hybridization buffer $(0.3 \mathrm{M} \mathrm{NaCl}, 10 \mathrm{mM} \mathrm{NaPO}, 10 \mathrm{mM}$ EDTA, $10 \mathrm{mM}$ Tris- $\mathrm{HCl}, 50 \%$ formamide, $10 \%$ dextran sulfate, $1 \mathrm{mg} / \mathrm{mL}$ tRNA, and $1 \mathrm{X}$ Denhartd's solution) with digoxigenin-labeled probes at $46^{\circ} \mathrm{C}$ overnight with $25 \mathrm{ng}$ of labeled probe for each section. After stringency washing in descending concentrations of standard saline citrate (SSC) and RNase A treatment $\left(10 \mu \mathrm{g} / \mathrm{mL}\right.$ at $37^{\circ} \mathrm{C}$ for $\left.15 \mathrm{~min}\right)$ for removal of unhybridized probe, probe hybridization was detected by binding of an anti-digoxigenin antibody (conjugated to alkaline phosphatase and visualized by an NBT/BICP substrate). In addition to hybridization with anti-sense probes, hybridization procedures for each of the 12 specimens were performed with omission of probes or with the sense probe for KGF as negative controls for confirmation of the specificity of the hybridization of the KGF anti-sense probe. Some specimens were also hybridized with an irrelevant anti-sense probe (for retinoic acid receptor $\alpha$ ) as a positive control.

\section{RT-PCR}

Reactions for each sample were performed with $1 \mu \mathrm{g}$ of total RNA and the GeneAmp RNA PCR Kit (Perkin Elmer, Norwalk, CT) used according to manufacturer's instructions. The primers used for amplification of KGF were the same as those previously described by Koos et al. (1993) which amplify a fragment of $266 \mathrm{bp}$ and consisted of: upper primer, $5^{\prime}$-TCT GTC GAA CAC AGT GGT ACC T, and lower primer, 5'-GTG TGT CCA TTT AGC TGA TGC AT. Primers for amplification of the KGF receptor were designed to amplify a 174-bp fragment and consisted of: upper primer, GGT TCT CAA CAC TCG GGG ATA, and lower primer, TCT CCT TTT CTC TTC CAG GCG. Negative controls for RT-PCR included reactions performed without the addition of RNA and without the reverse transcription step. RNA extracted from gingival fibroblasts, which have previously been demonstrated to express KGF (Gao and Mackenzie, 1996b), provided a positive control. PCR thermal cycling was carried out in a Strategene Robocycler 40 (La Jolla, CA) according to the following parameters: denaturation for $1 \mathrm{~min}$ at $95^{\circ} \mathrm{C}$, annealing for $1 \mathrm{~min}$ at $52^{\circ} \mathrm{C}$, and extension for $1 \mathrm{~min}$ at $72^{\circ} \mathrm{C}$. A denaturation step of $2 \mathrm{~min}$ and an extension step of $7 \mathrm{~min}$ were added to the initial and final cycles, respectively. Amplified products from 30 PCR cycles were separated by electrophoresis in $3 \%$ agarose gels, with DNA molecular marker VI (Boehringer Mannheim, Indianapolis, IN) used to provide size standards. The gels were stained with ethidium bromide and examined by ultraviolet transillumination. To confirm accuracy of sequence amplification with these primers, we cloned amplified fragments of the expected size for KGF and the KGFr into the PCR II vector (Invitrogene, San Diego, CA) and conducted sequence analysis using the fmol DNA Sequencing System 


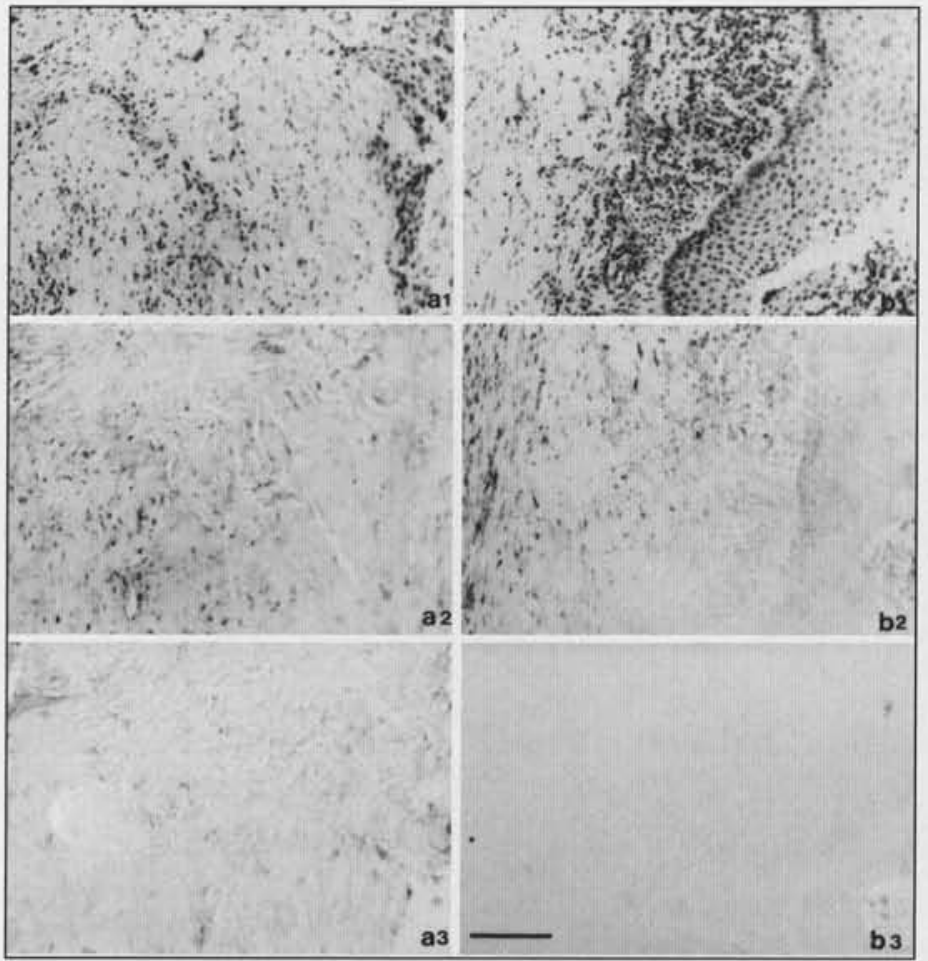

Figure 1. Sections of (a) a periapical granuloma and (b) a periapical cyst which were stained by H\&E (a1, b1) or processed for in situ hybridization by either the KGF anti-sense probe (a2, b2) or the sense probe $(\mathrm{a} 3, \mathrm{~b} 3)$. In the sections hybridized with the antisense probe, cells with a spindle-shaped, fibroblast-like morphology are stained positively for probe hybridization to KGF transcripts. Similar cell staining indicative of specific probe hybridization is not seen with the sense probe. (Scale $=200 \mu \mathrm{m}$ for all panels)

(Promega, Madison, WI) for comparison with published sequence data for KGF (Finch et al., 1989).

To provide a measure of the amount of KGF mRNA expressed, we also performed some reactions using a competitive PCR method. We constructed a cDNA internal standard, basically according to van den Heuvel et al. (1993), by PCR amplification of CDNA for the retinoic acid receptor $\alpha(\operatorname{RAR} \alpha)$, using a pair of oligonucleotide primers synthesized to include KGF primer tails. This produced a cDNA with short stretches of sequence corresponding to the upper and lower KGF primers positioned at each end of a 185-bp stretch of the RAR $\alpha$ cDNA, which acted as an irrelevant spacer sequence. Various amounts of the internal standard cDNA, ranging from 0.1 to $50 \mathrm{pg}$, were added to reaction tubes after the reverse transcription step was performed with $1 \mu \mathrm{g}$ of total RNA for each tissue sample. In the subsequent PCR reaction, the internal standard CDNA and the KGF cDNA derived from the RT step compete with similar efficiency for the KGF primers, but the amplified internal standard, which is $46 \mathrm{bp}$ smaller than the KGF product, is separated from the amplified KGF product in 3\% agarose gels. As the concentration of the internal standard decreases, the KGF band appears and increases. When the amount of PCR product for KGF matches that for the internal standard, the known concentration of the added internal standard in the reaction mixture is taken as being equivalent to that for the KGF cDNA.

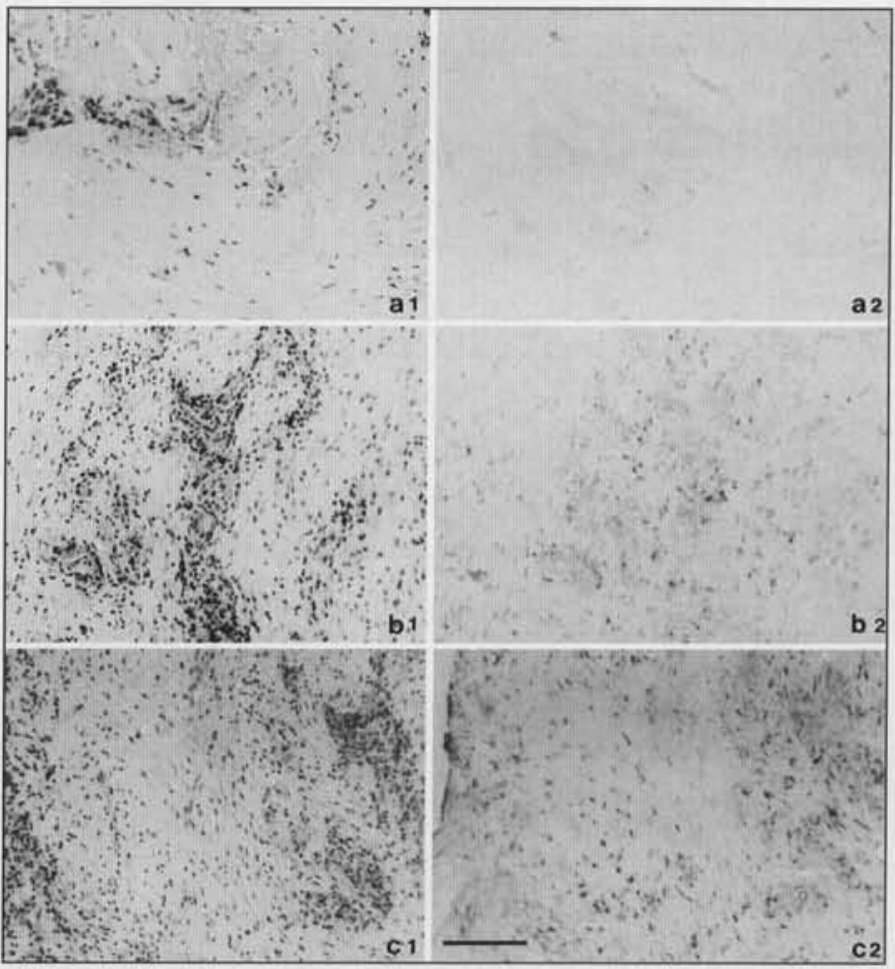

Figure 2. H\&E staining of sections of three periapical granulomas shows increasing levels of inflammatory cell infiltration from a1 to b1 to $c 1$, respectively. Adjacent sections of these specimens processed for in situ hybridization with KGF antisense probe (a2, b2, c2) show increased numbers of cells reacting positively for KGF transcripts as the inflammatory infiltrate increases. The proliferating epithelial elements identifiable by H\&E staining in b1 and $c 1$ are associated with cells expressing KGF mRNA, as seen in b2 and c2. (Scale $=200 \mu \mathrm{m}$ for all panels)

\section{Results}

The staining patterns observed in sections treated by in situ hybridization with the anti-sense probe for KGF indicated hybridization of probe to stromal cells in most periapical lesions. In situ hybridization procedures performed either without probes or with the KGF sense probe showed low levels of diffuse background staining but did not produce the clear patterns of cell staining indicative of specific hybridization. The RAR $\alpha$ anti-sense probe, used as a positive control for hybridization, showed clear staining indicative of specific hybridization; this was localized primarily in the epithelium.

Hybridization with the anti-sense KGF probe showed various numbers of positively stained cells which appeared to be stromal fibroblasts rather than inflammatory cells and to represent only a subpopulation of the stromal fibroblasts. Some cells showed weak staining, just above background levels, and others a more intense staining pattern (Fig. 1). Sections of normal PDL (and of 2 periapical granulomas that showed only mild inflammatory infiltration of the connective tissue) exhibited few cells expressing KGF transcripts. Greater numbers of positive cells were found in specimens showing dense inflammatory infiltrates, epithelial proliferation, or periapical cyst formation (Fig. 2). In periapical granulomas, KGF-expressing cells lay adjacent to inflammatory cell foci and close to proliferating epithelial 


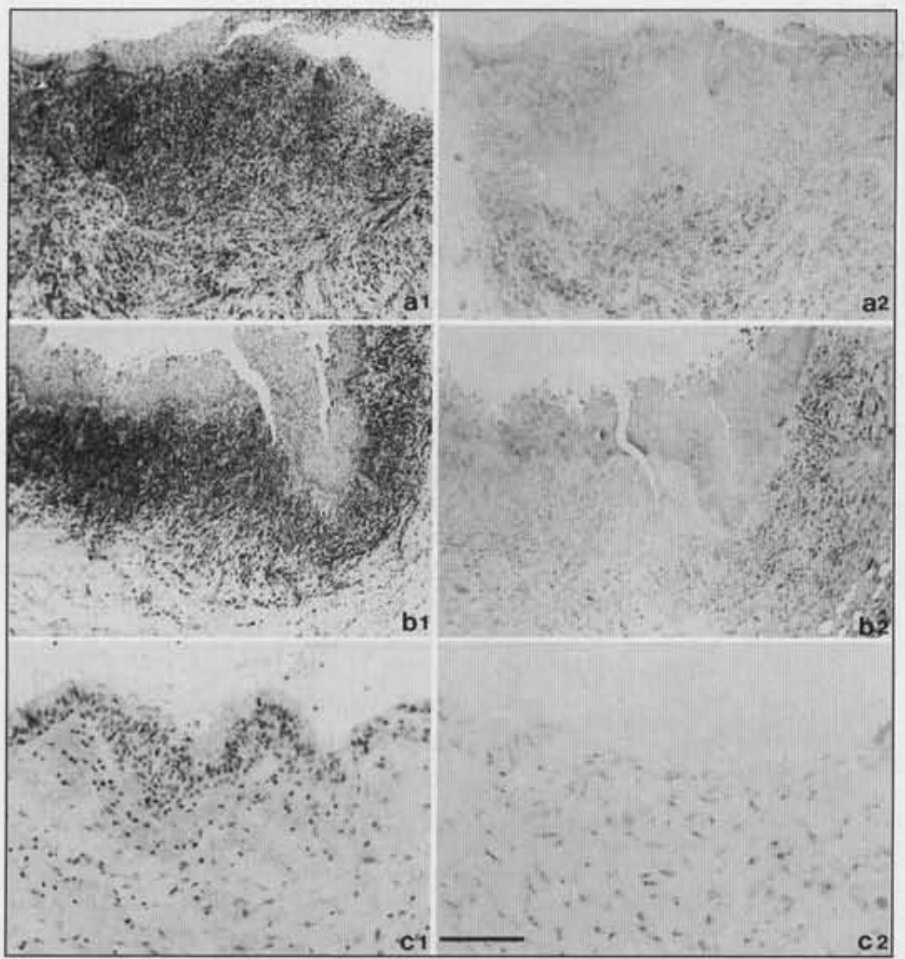

Figure 3. Sections of 3 periapical cysts stained with $H \& E$ (a1, b1, c1) or processed for in situ hybridization with the KGF anti-sense probe $(\mathrm{a} 2, \mathrm{~b} 2, \mathrm{c} 2)$. H\&E staining shows dense inflammatory infiltrates subjacent to the epithelial lining in a1 and b1 and a milder inflammatory infiltrate beneath the epithelium in c1. As shown in a2 and to the left of b2, cells expressing KGF mRNA tended to lie around, rather than within, dense infiltrates. However, as seen to the right in b2, strongly reactive cells sometimes lay within the infiltrate itself. Milder infiltrates (c1) tended to be associated with a more generalized pattern of KGF expression of the stromal fibroblasts. (Scale $=0.5 \mathrm{~mm}$ for panels $\mathrm{a}$ and $\mathrm{b}$ and $200 \mu \mathrm{m}$ for panel c)

elements. Typically, periapical cysts with a dense inflammatory infiltrate showed KGF-expressing cells at the periphery of the infiltrate, but cysts with milder or diffuse inflammatory infiltrates showed KGF-expressing cells close to the cystic lining (Fig. 3). Examination of H\&E-stained sections adjacent to those cut and processed for in situ hybridization allowed the tissue regions containing epithelium to be identified. KGF-positive cells could always be identified in those regions of periapical lesions where proliferating epithelium was present.

The periapical lesions evaluated by RT-PCR with specific primers for KGF showed products which appeared as a single band at a position corresponding to the expected length of 266 bp (Figs. 4a, 4b). Samples of normal PDL showed either no detectable PCR product or only a weak product band. Negative controls run without the addition of sample RNA or without the reverse transcription step failed to show product bands. A distinct KGF product band was detected for each of the 4 samples of periapical lesions (Fig. $4 \mathrm{~b})$ and also for RNA extracted from cultured gingival fibroblasts, which were used as a positive control. Assessment of the amount of products by competitive PCR (Fig. 4c) indicated that the PCR product from the target RNA (266-bp KGF fragment) increased as the PCR product

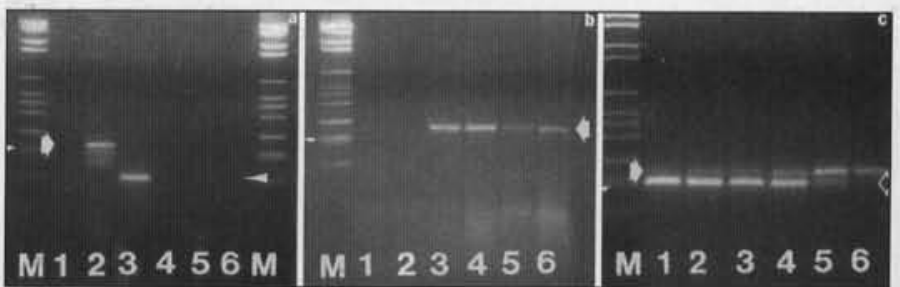

Figure 4. (a) RT-PCR amplification with RNA extracted from a periapical lesion (lanes 1-3) and from non-inflamed apical PDL (lanes 4 6). Lanes 1 and 4 show negative controls run without the reverse transcription step, lanes 2 and 5 show the amplification products with KGF primers, and lanes 3 and 6 the products with primers for the KGF receptor. Amplified transcripts of the appropriate size for KGF (large arrow) and the KGF receptor (small arrow) were produced from the lesional RNA (lanes 2 and 3) but under the same conditions were not detectable for RNA extracted from healthy PDL (lanes 5 and 6). (b) RT/PCR amplification of KGF mRNA from 2 samples of uninflamed PDL and from 4 samples of periapical lesions (lanes 3-6). No product was amplified from normal PDL (lanes 1 and 2), but, from each of the 4 lesional samples, product of the appropriate size for KGF was amplified in various amounts. (c) Competitive PCR (see text) with RNA extracted from a periapical lesion to which an internal standard was added in decreasing quantities from 50 to $0.1 \mathrm{pg}$ (lane 1 to lane 6). The relative densities of the product bands for the internal standard and for KGF reverse between lane 4 (1-pg internal standard) and lane $5(0.5 \mathrm{pg})$. The solid arrow indicates bands for the KGF product, and the hollow arrow indicates product derived from the internal standard. For all panels, the small arrow indicates the 234-bp marker band. $M=c D N A$ size marker.

from the internal standard ( $241 \mathrm{bp}$ ) decreased, and also that the equivalency point between the two products occurred at approximately from 0.5 to $1 \mathrm{pg}$ of added internal standard. RT-PCR with primers for the KGFr resulted in amplified product of the expected size of $175 \mathrm{bp}$ from RNA extracted from the periapical lesions but not from the specimens of normal periodontium (Fig. 4a). The accuracy of PCR amplification for both KGF and the KGFr was confirmed by the exact match with published data for the KGF and KGFr sequences (Finch et al., 1989; Miki et al., 1992) found for samples of amplified product that were cloned into a PCR II vector for sequencing.

\section{Discussion}

The results of the in situ hybridization studies indicate that KGF mRNA is expressed more frequently and more strongly by the stromal cells of periapical lesions than those of normal periodontal ligament. These observations are also supported by the results of the RT-PCR and competitive PCR which indicated that levels of KGF expression in periapical lesions are quite high and similar to those of gingival fibroblasts in vitro (Gao and Mackenzie, 1996b). It thus appears that KGF may thus play a role in the epithelial proliferation associated with the pathogenesis of periapical lesions. Although derived from relatively few samples, and with methods of analysis that did not permit quantification for statistical analysis, the consistency of these observations suggests a general correlation among inflammation, epithelial proliferation, and KGF expression.

KGF was initially isolated as a product of human 
fibroblasts that stimulates epithelial growth (Rubin et al., 1989). The subsequent cloning and sequencing of KGF cDNA indicated that it codes for a 194-amino-acid polypeptide with homology to the heparin-binding family of fibroblast growth factors, and that KGF contains a hydrophobic signal sequence in the $\mathrm{N}$-terminal region indicating that it is a secreted protein (Finch et al., 1989). KGF mRNA and the biologically active peptide are expressed in vitro by various human fibroblast cell lines, and it is suggested that KGF acts specifically as a paracrine mediator of epithelial proliferation (Finch et al., 1989), a concept that is supported by the expression of the KGF receptor only on epithelial cells (Aaronson et al., 1991) and by the observed mitogenicity of KGF for epithelial but not stromal cells (Le Panse et al., 1994). Further, Guo et al. (1993), using a human keratin 14 promoter to target expression of human KGF cDNA to stratified squamous epithelia in transgenic mice, have demonstrated that overexpression of KGF is associated with proliferative epithelial changes in vivo.

In view of these roles of KGF in the development, growth, and differentiation of epithelia, the present finding that KGF transcripts are expressed at higher levels and by more stromal cells in periapical granulomas and cysts than in healthy PDL is an interesting one. The rests of Malassez are not normally proliferative, but our present observations, and those of previous studies (Summers, 1974), indicate that proliferating epithelium is commonly found in inflammatory periapical lesions. Inflammation is associated with the generation of multiple interacting cytokine networks, and some of the cytokines produced-such as epidermal growth factor (EGF), transforming growth factor $\alpha$ (TGF $\alpha)$, tumor necrosis factor, and interleukin-6-are known to have stimulatory effects on the proliferation of other epithelia in vitro (Luger and Schwartz, 1994). Rests of Malassez express some receptors-for example, the EGF/TGF $\alpha$ receptor (Thesleff, 1987)-through which such cytokines could act, and possibly other factors (such as interleukins and interferon) may play a role in this process (Brown and Smith, 1991). However, the actual magnitude of direct in vivo epithelial responses to inflammatory cytokines remains uncertain. The recent findings concerning the actions and patterns of induction of KGF suggest that the major effect of such cytokines may be an indirect one via their induction of KGF expression. Examination of the mechanisms of up-regulation of KGF expression in fibroblasts indicates that it is induced by exposure to growth factors such as interleukin $1 \alpha$ and $\beta$, TNF $\alpha$, and PDGF (Brauchle et al., 1994; Chedid et al., 1994), which are produced both by inflammatory cells and by epithelia. The expression of KGF in subepithelial fibroblasts appears to be normally signaled by products of the epithelium itself (Smola et al., 1993).

The observed relationship of KGF-expressing cells to apical inflammatory infiltrates suggests that the inflammatory process induces elevated expression of KGF by periodontal fibroblasts. The association of such KGF expression with proliferating epithelium suggests that KGF may be responsible for activating and maintaining proliferation of the epithelial rests. The detection of message for the KGF receptor in periapical lesions suggests that proliferating periapical epithelia are able to respond to the KGF stimulus. The present study examined KGF expression only at the RNA message level; whether the biologically active peptide is also present in periapical lesions needs to be investigated. Nevertheless, other studies have shown that the expression of KGF message correlates with the production and secretion of KGF (Brauchle et al., 1994; Chedid et al., 1994). Our preliminary observations point to an interesting indirect mechanism by which common inflammatory cytokines could stimulate proliferation and growth of the rests of Malassez by inducing fibroblast production of a potent epithelial mitogen.

\section{Acknowledgments}

This work was supported by NIH/NIDR grant \#R01 DE010317.

\section{References}

Aaronson SA, Bottaro DP, Miki T, Ron D, Finch PW, Fleming $\mathrm{TP}$, et al. (1991). Keratinocyte growth factor: A fibroblast growth factor family member with unusual target cell specificity. Ann NY Acad Sci 638:62-77.

Brauchle M, Angermeyer K, Hubner G, Werner S (1994). Large induction of keratinocyte growth factor expression by serum growth factors and pro-inflammatory cytokines in cultured fibroblasts. Oncogene 9:3199-3204.

Brown RM, Smith AJ (1991). Pathogenesis of odontogenic cysts. In: Investigative pathology of the odontogenic cysts. Brown RM, editor. Boston: CRC Press, pp. 87-109.

Chedid M, Rubin JS, Csaky KG, Aaronson SA (1994). Regulation of keratinocyte growth factor gene expression by interleukin 1. J Biol Chem 269:10753-10757.

Cymerman JJ, Cymerman DH, Walters J, Nevins AJ (1984). Human T lymphocyte subpopulations in chronic periapical lesions. J Endodont 10:9-11.

Finch PW, Rubin JS, Miki T, Ron D, Aaronson SA (1989). Human KGF is FGF-related with properties of a paracrine effector of epithelial cell growth. Science 245:752-755.

Gao Z, Mackenzie IC, Williams DM, Cruchley AT, Leigh I, Lane EB (1988a). Patterns of keratin-expression in rests of Malassez and periapical lesions. J Oral Pathol 17:178-185.

Gao Z, Mackenzie IC, Rittman BR, Korszun A-K, William DM, Cruchley AT (1988b). Immunocytochemical examination of immune cells in periapical granulomata and odontogenic cysts. J Oral Pathol 17:84-90.

Gao Z, Mackenzie IC (1996a). Influence of retinoic acid on the expression of cytokeratins, vimentin and ICAM-1 in human gingival epithelia in vitro. J Periodont Res 31:81-89.

Gao Z, Mackenzie IC (1996b). Keratinocyte growth factor expression in human gingival fibroblasts and stimulation of in vitro gene expression by retinoic acid. J Periodont Res (in press).

Grupe HE, Ten Cate AR, Zander HA (1967). A histochemical and radiological study of in vitro and in vivo human epithelial cell rest proliferation. Arch Oral Biol 12:1321-1329.

Guo L, Yu QC, Fuchs E (1993). Targeting expression of keratinocyte growth factor to keratinocytes elicits striking 
changes in epithelial differentiation in transgenic mice. EMBO / 12:973-986.

Housley RM, Morris CF, Boyle W, Ring B, Biltz R, Tarpley JE, et al. (1994). Keratinocyte growth factor induces proliferation of hepatocytes and epithelial cells throughout the rat gastrointestinal tract. J Clin Invest 94:1764-1777.

Imagawa W, Cunha GR, Young P, Nandi S (1994). Keratinocyte growth factor and acidic fibroblast growth factor are mitogens for primary cultures of mammary epithelium. Biochem Biophys Res Commun 204:1165-1169.

Koos RD, Banks PK, Inkster SE, Yue W, Brodie AM (1993). Detection of aromatase and keratinocyte growth factor expression in breast tumors using reverse transcriptionpolymerase chain reaction. I Steroid Biochem Molec Biol 45:217-225.

Le Panse R, Mitev V, Lebreton C, Coulomb B (1994). Modulation of epidermal growth factor and keratinocyte growth factor effects on human keratinocyte growth by protein kinase $C$ inhibitor GF109203X: Comparision to fibroblast growth modulation. Biochem Biophys Res Commun 204:1081-1087.

Luger, Schwartz (1994). Epidermal growth factors and cytokines. New York: Marcel Dekker, Inc., pp. 76-84.

Marchese C, Rubin J, Ron D, Faggioni A, Torris MR, Messina A, et al. (1990). Human keratinocyte growth factor activity on proliferation and differentiation of human keratinocytes: Differentiation response distinguishes KGF from EGF family. J Cell Physiol 144:326-332.

Miki T, Bottaro DP, Fleming TP, Smith CL, Burgess WH, Chan AM, et al. (1992). Determination of ligand-binding specificity by alterative splicing: Two distinct growth factor receptors encoded by a single gene. Proc Natl Acad Sci USA 89:246-250.

Rubin JS, Osada H, Finch PW, Taylor WG, Rudikoff S, Aaronson SA (1989). Purification and characterization of a newly identified growth factor specific for epithelial cells.
Proc Natl Acad Sci USA 86:802-806.

Shear M (1985). Cysts of the jaw: Recent advances. J Oral Pathol 14:43-59.

Smola H, Thiekotter G, Fusenig NE (1993). Mutual induction of growth factor gene expression by epidermal-dermal cell interaction. J Cell Biol 122:417-429.

Stern MH, Dreizen S, Mackler BF, Levy BM (1982). Isolation and characterization of inflammatory cells from human periapical granuloma. J Dent Res 61:1408-1412.

Summers L (1974). The incidence of epithelium in periapical granulomas and mechanism of cavitation in apical dental cysts in man. Arch Oral Biol 19:1177-1180.

Ten Cate AR (1972). The epithelial cell rests of Malassez and the genesis of the dental cyst. Oral Surg Oral Med Oral Pathol 34:956-964.

Thesleff I (1987). Epithelial cell rests of Malassez bind epidermal growth factor intensely. J Periodont Res 22:419-421.

Torabinejad M, Kettering JD (1985). Identification and relative concentration of $\mathrm{B}$ and $\mathrm{T}$ lymphocytes in human chronic periapical lesions. J Endodont 11:122-125.

van den Heuvel JP, Tyson FL, Bell DA (1993). Construction of recombinant RNA templates for use as internal standards in quantitative RT-PCR. Biotechniques 14:395-398.

Werner S, Peters KG, Longaker MT, Fuller-Pace F, Banda MJ, Williams LT (1992). Large induction of keratinocyte growth factor expression in the dermis during wound healing. Proc Natl Acad Sci USA 89:6896-6900.

Werner S, Smola H, Liao X, Longaker MT, Krieg T, Hofschneider PH, et al. (1994). The function of KGF in morphogenesis of epithelium and the re-epithelialization of wounds. Science 266:819-822.

Yi ES, Yin S, Harclerode DL, Bedoya A, Bikhazi NB, Housley RM, et al. (1994). Keratinocyte growth factor induces pancreatic ductal epithelial proliferation. Am J Pathol 145:80-85. 